Original Research Paper

\title{
On Bayesian Premium Estimators for Gamma Lindley Model under Squared Error Loss Function and Linex Loss Function
}

\author{
Ahmed Sadoun, Halim Zeghdoudi, \\ Fatma Zohra Attoui and Mohamed Riad Remita \\ LaPS Laboratory, Badji-Mokhtar University - Annaba, Algeria
}

Article history

Received: 8-3-2017

Revised: 10-6-2017

Accepted: $15-9-2017$

Corresponding Author: Halim Zeghdoudi, LaPS Laboratory, Badji

Mokhtar University-Annaba,

Algeria

Email: hzeghdoudi@yahoo.fr

\begin{abstract}
We consider the Gamma Lindley distribution $(\mathrm{GaL})$ as the conditional distribution of $(X \mid \theta, \gamma)$, we focus on the estimation of the Bayesian premium under squared error loss function (symmetric) and linearexponential (Linex) loss function (asymmetric), using informative priors (the Gamma prior). Because of its difficulty and non-linearity, we use a numerical approximation for computing the Bayesian premium. Finally, a simulation and comparative study with varying sample sizes are given.
\end{abstract}

Keywords: Bayesian Premium, Gamma Lindley Distribution, Gamma Distribution, Loss Function, Entropy, Linex

\section{Introduction}

The credibility theory is one of the important quantitative techniques in actuarial science that allow insurance companies to perform an experience assessment (adjusting future premiums based on past experience). We deal the Bayesian premium estimator developed by Bailey (1950), considering Gamma Lindley distribution as a claim distribution which is a popular tool in credibility theory. The Gamma Lindley distribution was first published in the literature from 2016 by Zeghdoudi and Nedjar. This distribution is based on mixtures of the ordinary Gamma $(2, \theta)$ and Lindley $(\theta)$ distributions, which is one of the distributions that models survival analysis data and insurance. There are a lot of papers study the new distributions to know: Lindley (1958), Sankaran (1970), Ghitany and Al-Mutairi (2009), Shanker et al. (2013), Ghitany et al. (2008), Zeghdoudi and Lazri (2016) Zeghdoudi and Nedjar (2016; 2016a; 2016b; 2017; 2017a). Recently, Krishna and Kumar (2011) used the Bayesian approach and the maximum probability for an incomplete set of data using various loss functions. On the other hand, Dey et al. (1992, 1999), Ibrahim et al. (2012), Ali et al. (2013) and Metiri et al. (2016) investigated the effect of certain loss functions on Bayes Estimate and the subsequent risk for Lindley's using noninformative and informative priors.

The structure of the paper is as follows: Section 2 is devoted to describing the model, the Maximum Likelihood Estimators (MLE), asymptotic confidence intervals and Bayes Estimators (BE). In Section 3, we give the derivation of this estimator under squared error loss and linear-exponential loss with informative prior using numerical approximation (Lindley approximation). Finally, we evaluate the Bayesian premium estimators under the above loss functions; a simulation using Monte Carlo method and mean squared error technique are given.

\section{Model and Bayesian Inferences}

In this section, we consider the MLE of the parameters, and we suppose that, $X_{1: n}, X_{2: n}, \ldots, X_{n: n}$, are independent observations in presence of Type-I hybrid censored samples from $\operatorname{GaL}(\theta, \gamma)$. Thus, the likelihood functions for this case are:

$$
L(\theta, \gamma)=\frac{\theta^{2 n}}{[\gamma(\theta+1)]^{n}} \prod_{i=1}^{n}\left[(\gamma+\gamma \theta-\theta) x_{i}+1\right] e^{-\theta\left(\sum_{i=1}^{n} x_{i}\right)}
$$

The Log likelihood function is:

$$
\begin{aligned}
& \log L(x \mid \theta, \gamma)=2 n \log \theta-n \log \gamma-n \log (\theta+1) \\
& +\sum_{i=1}^{n} \log \left[(\gamma+\gamma \theta-\theta) x_{i}+1\right]-\theta \sum_{i=1}^{n} x_{i}
\end{aligned}
$$

Now, we can use Newton-Raphson (N-R) method of the following normal equations:

$$
\begin{aligned}
& \frac{2 n}{\theta}-\frac{n}{(\theta+1)}-\sum_{i=1}^{n} x_{i}+(\gamma-1) \sum_{i=1}^{n} \frac{x_{i}}{\left[(\gamma+\gamma \theta-\theta) x_{i}+1\right]}=0, \\
& \frac{-n}{\gamma}+(\theta+1) \sum_{i=1}^{n} \frac{x_{i}}{\left[(\gamma+\gamma \theta-\theta) x_{i}+1\right]}=0
\end{aligned}
$$


Using some regularity conditions, we find the MLEs $(\hat{\theta}, \hat{\gamma})$ is approximately bi-variate normal with mean $(\hat{\theta}, \hat{\gamma})$ and covariance matrix:

$$
I^{-1}(\hat{\theta}, \hat{\gamma}) \text { i.e. }(\hat{\theta}, \hat{\gamma}) \sim N_{2}\left([\hat{\theta}, \hat{\gamma}]^{\prime}, I^{-1}(\hat{\theta}, \hat{\gamma})\right)
$$

where, $I=(\hat{\theta}, \hat{\gamma})$ is the observed Fisher Information Matrix (FIM) and defined as:

$$
I(\hat{\theta}, \hat{\gamma})=\left[\begin{array}{cc}
-\frac{\partial^{2} \log L}{\partial \theta^{2}} & -\frac{\partial^{2} \log L}{\partial \theta \partial \gamma} \\
-\frac{\partial^{2} \log L}{\theta \partial \gamma} & -\frac{\partial^{2} \log L}{\partial \gamma^{2}}
\end{array}\right]_{(\hat{\theta}, \hat{\gamma})}
$$

The diagonal elements of inverse FIM $I^{-1}(\hat{\theta}, \hat{\gamma})$ provides the asymptotic variances for the parameters $\theta$ and $\gamma$. Then two-side $100(1-\gamma) \%$ normal approximation confidence interval of $\theta$ and $\gamma$ can be defined as $\left\{\hat{\theta} \pm Z_{\frac{\alpha}{2}} \sqrt{\operatorname{var}(\hat{\theta})}\right\}$ and $\left\{\hat{\gamma} \pm Z_{\frac{\alpha}{2}} \sqrt{\operatorname{var}(\hat{\gamma})}\right.$.

\section{Bayesian Inferences for the Parameters}

We obtain in this section the BE of the parameters $\theta$ and $\gamma$. We need in Bayesian analysis to specify prior distribution for the parameters, for that, we take two independent gamma priors such as $\operatorname{Gamma}(a, b)$ as a prior of $\theta$ and $\operatorname{Gamma}(c, d)$ consider as a prior of $\gamma$ where $a, b, c$ and $d$ are non negative hyper-parameters:

$$
\begin{aligned}
& g(\theta)=\frac{b^{a}}{\Gamma(a)} \theta^{a-1} e^{-b \theta} ; \theta, a, b>0 \\
& g(\gamma)=\frac{d^{c}}{\Gamma(c)} \gamma^{c-1} e^{-d \gamma} ; \gamma, a, b>0
\end{aligned}
$$

Now, the joint prior distribution for $\theta$ and $\gamma$ is given as:

$$
\pi(\theta, \gamma) \propto \theta^{a-1} \gamma^{c-1} e^{-(b \theta+d \gamma)} ; a, b, c, d>0
$$

And, the joint posterior is given as:

$$
p(\theta, \gamma \mid x)=R^{-1} \theta^{2 n+a-1} \gamma^{c-n-1} e^{-\left[d \gamma+\theta\left(\sum_{i=1}^{n} x_{i}+b\right)\right]} P(\theta, \gamma) d \theta d \gamma
$$

where, $R$ and $P(\theta, \gamma)$ are interpreted as:

$$
\begin{gathered}
R=\iint_{\theta \gamma} \theta^{2 n+a-1} \gamma^{c-n-1} e^{-\left[d \gamma+\theta\left(\left(\sum_{i=1}^{n} x_{i}+b\right)\right)\right]} P(\theta, \gamma) d \theta d \gamma \\
P(\theta, \gamma)=\frac{\prod_{i=1}^{n}\left[(\gamma+\gamma \theta-\theta) x_{i}+1\right]}{(\theta+1)^{n}}
\end{gathered}
$$

\section{Around Lindley's Approximation}

We can see now that the posterior distribution of $(\theta, \gamma)$ takes ratios form that involves an integration in the denominator. Moreover, we notice that cannot reduced to a closed form. There is a simplest methods proposed to approximate the ratio of the integrals of the above form, is Lindley's Approximation (1980). Several studies have used this. Several studies have used this approximation for obtaining the $\mathrm{BE}$ for some distributions (Howlader and Hossain (2002) and Jaheen (2005)). Using the Lindley's approximation technique for the estimation of the $\theta$ and $\gamma$, where the posterior expectation is:

$$
(x)=E(\theta, \gamma x)=\frac{\int u(\gamma, \theta) e^{L(\gamma, \theta)+G(\gamma, \theta)} d(\gamma, \theta)}{\int e^{L(\gamma, \theta)+G(\gamma, \theta)} d(\gamma, \theta)}
$$

$u(\theta, \gamma)$ is a function of $\theta$ and $\gamma, L(\theta, \gamma) \log$-Likelihood function and $G(\theta, \gamma) \log$ of joint prior density.

If ML estimates of the parameters are available and $n$ are sufficiently large then the above ratio of the integral can be approximated as:

$$
\begin{aligned}
& I(x)=u(\hat{\theta}, \hat{\gamma})+\frac{1}{2}\left[\begin{array}{l}
\left(\hat{u}_{\gamma \gamma}+2 \hat{u}_{\gamma} \hat{p}_{\gamma}\right) \hat{\sigma}_{r \gamma}+\left(\hat{u}_{\theta \gamma}+2 \hat{u}_{\theta} \hat{p}_{\gamma}\right) \hat{\sigma}_{\theta \gamma} \\
+\left(\hat{u}_{\gamma \theta}+2 \hat{u}_{\gamma} \hat{p}_{\theta}\right) \hat{\sigma}_{\gamma \theta}+\left(\hat{u}_{\theta \theta}+2 \hat{u}_{\theta} \hat{p}_{\theta}\right) \hat{\sigma}_{\theta \theta}
\end{array}\right]+\frac{1}{2} \\
& {\left[\begin{array}{l}
\left(\hat{u}_{\gamma} \hat{\sigma}_{\gamma \gamma}+\hat{u}_{\theta} \hat{\sigma}_{\gamma \theta}\right)\left(\hat{L}_{r y} \hat{\sigma}_{r \gamma}+\hat{L}_{\gamma \theta \gamma} \hat{\sigma}_{\gamma \theta}+\hat{L}_{\theta \gamma} \hat{\sigma}_{\theta \gamma}+\hat{L}_{\theta \theta} \hat{\sigma}_{\theta \theta}\right) \\
+\left(\hat{u}_{\gamma} \hat{\sigma}_{\theta \gamma}+\hat{u}_{\theta} \hat{\sigma}_{\theta \theta}\right)\left(\hat{L}_{\theta \gamma} \hat{\sigma}_{\theta \theta}+\hat{L}_{\gamma \theta \theta} \hat{\sigma}_{\gamma \theta}+\hat{L}_{\theta \gamma \theta} \hat{\sigma}_{\theta \gamma}+\hat{L}_{\theta \theta \theta} \hat{\sigma}_{\theta \theta}\right)
\end{array}\right]}
\end{aligned}
$$

where, $\hat{\theta}$ and $\hat{\gamma}$ are the MLE of $\theta$ and $\gamma$ (see Lindley (1980)):

$$
\begin{aligned}
& \hat{u}_{\theta}=\frac{\partial u(\hat{\theta}, \hat{\gamma})}{\partial \hat{\theta}}, \hat{u}_{\gamma}=\frac{\partial u(\hat{\theta}, \hat{\gamma})}{\partial \hat{\gamma}}, \hat{u}_{\theta \gamma}=\frac{\partial u(\hat{\theta}, \hat{\gamma})}{\partial \hat{\theta} \theta \hat{\gamma}}, \hat{u}_{\gamma \theta}=\frac{\partial u(\hat{\theta}, \hat{\gamma})}{\partial \hat{\gamma} \partial \hat{\theta}} \\
& \hat{u}_{\theta \theta}=\frac{\partial^{2} u(\hat{\theta}, \hat{\gamma})}{\partial \hat{\theta}^{2}}, \hat{u}_{\gamma \gamma}=\frac{\partial^{2} u(\hat{\theta}, \hat{\gamma})}{\partial \hat{\gamma}^{2}}, \hat{p}_{\theta}=\frac{\partial G(\hat{\theta}, \hat{\gamma})}{\partial \hat{\theta}}, \hat{p}_{\gamma}=\frac{\partial G(\hat{\theta}, \hat{\gamma})}{\partial \hat{\gamma}}, \\
& \hat{L}_{\theta \theta}=\frac{\partial^{2} L(\hat{\theta}, \hat{\gamma})}{\partial \hat{\theta}^{2}}, \hat{L}_{\gamma \gamma}=\frac{\partial^{2} L(\hat{\theta}, \hat{\gamma})}{\partial \hat{\gamma}^{2}}, \hat{L}_{\theta \theta \theta}=\frac{\partial^{3} L(\hat{\theta}, \hat{\gamma})}{\partial \hat{\theta}^{3}}, \\
& \hat{L}_{\theta \theta \gamma}=\frac{\partial^{3} L(\hat{\theta}, \hat{\gamma})}{\partial \theta \partial \theta \partial \gamma}, \hat{L}_{\gamma \theta}=\frac{\partial^{3} L(\hat{\theta}, \hat{\gamma})}{\partial \gamma \partial \gamma \partial \theta}, \hat{L}_{\gamma \theta \gamma}=\frac{\partial^{3} L(\hat{\theta}, \hat{\gamma})}{\partial \gamma \partial \theta \partial \gamma} \\
& \hat{L}_{\theta \gamma}=\frac{\partial^{3} L(\hat{\theta}, \hat{\gamma})}{\partial \theta \partial \gamma \partial \gamma}, \hat{L}_{\gamma \theta \theta}=\frac{\partial^{3} L(\hat{\theta}, \hat{\gamma})}{\partial \gamma \partial \theta \partial \theta}
\end{aligned}
$$
where:

We replace (2.4) $(p(\theta, \gamma \mid X))$ in the expression of $I(x)$,

$$
\begin{aligned}
u(\theta, \gamma) & =\gamma, \\
L(\theta, \gamma) & =2 n \log \theta-n \log \gamma-n \log (\theta+1) \\
& +\sum_{i=1}^{n} \log \left[(\gamma+\gamma \theta-\theta) x_{i}+1\right]-\theta \sum_{i=1}^{n} x_{i}, \\
G(\theta, \gamma) & =(a-1) \log \theta+(c-1) \log \gamma-(b \theta+d \gamma)
\end{aligned}
$$


It may verified that:

$$
\begin{aligned}
& u_{\gamma}=1, u_{\gamma \gamma}=u_{\gamma \theta}=u_{\theta \theta}=u_{\theta \gamma}=0, p_{\theta}=\frac{a-1}{\theta}-b, p_{\gamma}=\frac{c-1}{\gamma}-d, \\
& L_{\theta}=\frac{2 n}{\theta}-\frac{n}{(\theta+1)}+\sum_{i=1}^{n} \frac{(\gamma-1) x_{i}}{\left[(\gamma+\gamma \theta-\theta) x_{i}+1\right]}-\sum_{i=1}^{n} x_{i}, \\
& L_{\theta \theta}=\frac{-2 n}{\theta^{2}}+\frac{n}{(\theta+1)^{2}}-\sum_{i=1}^{n} \frac{\left[(\gamma-1) x_{i}\right]^{2}}{\left[(\gamma+\gamma \theta-\theta) x_{i}+1\right]^{2}} \text {, } \\
& L_{\theta \theta \theta}=\frac{4 n}{\theta^{3}}-\frac{2 n}{(\theta+1)^{3}}+\sum_{i=1}^{n} \frac{2\left[(\gamma-1) x_{i}\right]^{3}}{\left[(\gamma+\gamma \theta-\theta) x_{i}+1\right]^{3}}, \\
& L_{\theta \theta \gamma}=L_{\gamma \theta \theta}=\sum_{i=1}^{n} \frac{2 x_{i}^{2}(1-\gamma)\left(1+x_{i}\right)}{\left[(\gamma+\gamma \theta-\theta) x_{i}+1\right]^{3}} \text {, } \\
& L_{\theta \gamma \gamma}=L_{\gamma \theta}=-\sum_{i=1}^{n} \frac{2 x_{i}^{2}(1+\theta)\left(1+x_{i}\right)}{\left[(\gamma+\gamma \theta-\theta) x_{i}+1\right]^{3}}, \\
& L_{\theta \gamma}=L_{\gamma \theta}=\sum_{i=1}^{n} \frac{x_{i}\left(1+x_{i}\right)}{\left[(\gamma+\gamma \theta-\theta) x_{i}+1\right]^{2}}, \\
& L_{\gamma}=\frac{-n}{\gamma}+\sum_{i=1}^{n} \frac{(1+\theta) x_{i}}{\left[(\gamma+\gamma \theta-\theta) x_{i}+1\right]} \text {, } \\
& L_{\gamma \gamma}=\frac{n}{\gamma^{2}}-\sum_{i=1}^{n} \frac{\left[(1+\theta) x_{i}\right]^{2}}{\left[(\gamma+\gamma \theta-\theta) x_{i}+1\right]^{2}} \text {, } \\
& L_{m \gamma}=\frac{-2 n}{\gamma^{3}}+\sum_{i=1}^{n} \frac{2\left[(1+\theta) x_{i}\right]^{3}}{\left[(\gamma+\gamma \theta-\theta) x_{i}+1\right]^{3}},
\end{aligned}
$$

Again, because $\theta$ and $\gamma$ are independent $(X \mid \theta, \gamma), \sigma_{\gamma \theta}=$ 0 for $\theta \neq \gamma$ and $\sigma_{r \gamma}=-\frac{1}{L_{\gamma \gamma}} ; \sigma_{\theta \theta}=-\frac{1}{L_{\theta \theta}}$. The approximate BE of $\theta$ under SELF after evaluation of all u-terms, Lterms, and p-terms at the point $(\hat{\theta}, \hat{\gamma})$ is:

$$
\hat{\theta}_{B}=\hat{\theta}+\hat{u}_{\theta} \hat{p}_{\theta} \hat{\sigma}_{\theta \theta}+0.5\left[\left(\hat{u}_{\theta} \hat{\sigma}_{\theta \theta}\right)\left(\hat{L}_{\theta \gamma \gamma} \hat{\sigma}_{r \gamma}+\hat{L}_{\theta \theta \theta} \hat{\sigma}_{\theta \theta}\right)\right]
$$

Thus, the BE of $\gamma$ under SELF is:

$$
\begin{aligned}
& u_{\gamma}=1, u_{\gamma \gamma}=u_{\gamma \theta}=u_{\theta \theta}=u_{\theta \gamma}=0 \text { and } \\
& \hat{\gamma}_{B}=\hat{\gamma}+\hat{u}_{\gamma} \hat{p}_{\gamma} \hat{\sigma}_{\gamma \gamma}+0.5\left[\left(\hat{u}_{\gamma} \hat{\sigma}_{\gamma \gamma}\right)\left(\hat{L}_{r \gamma} \hat{\sigma}_{\gamma \gamma}+\hat{L}_{\theta \theta \gamma} \hat{\sigma}_{\theta \theta}\right)\right]
\end{aligned}
$$

\section{Derivation of Bayesian Premiums}

To obtain Bayesian premium estimators, we must take $\theta$ and $\gamma$ the real-valued random variables with probability density function $\pi(\theta, \gamma)$. Recall that the conditional distribution of $(X \mid \theta, \gamma)$ is the Gamma Lindley distribution and the distribution of $\theta$ and $\gamma$ is assumed to be known in the present section. $p(\theta, \gamma \mid X)$ is the posterior distribution of $\theta$ and $\gamma$ gave the data. This section considers estimation of the Bayesian premium $p^{B}$ based on the above-mentioned priors and loss functions.

On Bayesian Premium Estimators under Squared Error Loss Function

The squared error loss function denotes the punishment in using $\hat{\beta}$ to estimate $\beta$ and is given by $L(\hat{\beta}, \beta)=(\hat{\beta}-\beta)^{2}$ proposed by Legendre (1805) and Bühlmann Straub (1996) to develop least squares theory. The squared error loss function is symmetric in nature i.e. it gives equal weight age to both over and under estimation. In the actuarial literature, we write:

$$
L\left(P_{S E L F}^{B}, \mu(\theta, \gamma)\right)=\left(P_{S E L F}^{B}-\mu(\theta, \gamma)\right)^{2}
$$

The Bayesian premium $P_{S E L F}^{B}$ is the estimator of $\mu(\theta, \gamma)$, it is to be chosen such that the posterior expectation of the squared error loss function:

$$
\begin{aligned}
& E\left[L\left(P_{S E L F}^{B}, \mu(\theta, \gamma)\right)\right] \\
& =\int_{0}^{\infty} \int_{0}^{\infty} L\left(P_{S E L F}^{B}, \mu(\theta, \gamma)\right) P(\theta, \gamma \mid x) d \theta d \gamma \\
& =\int_{0}^{\infty} \int_{0}^{\infty}\left(P_{S E L F}^{B}-\mu(\theta, \gamma)\right)^{2} P(\theta, \gamma \mid x) d \theta d \gamma
\end{aligned}
$$

is minimum:

$$
P_{S E L F}^{B}=E[\mu(\theta, \gamma) \mid X]=\int_{0}^{\infty} \int_{0}^{\infty} \mu(\theta, \gamma) P(\theta, \gamma \mid x) d \theta d \gamma
$$

is the individual premium.

\section{Posterior Distribution using the Gamma Prior}

The informative prior provides more information than the non-informative priors, which play a important role in statistical inference and science actuarial. Therefore, the analysis using these prior more accurate and informative than classical approach. The Gamma prior is a good life distribution may be convenient for representing uncertainly in a current estimate of the claim frequency rate. This distribution is over the whole positive range from 0 to $+\infty$. It is given as:

$\pi(\theta, \gamma) \propto \theta^{a-1} \gamma^{c-1} e^{-(b \theta+d \gamma)} ; a, b, c, d>0$

where, $a, b, c$ and $d$ are the hyper-parameters.

Where:

$$
u(\theta, \gamma)=\mu(\theta, \gamma)=\frac{2 \gamma(1+\theta)-\theta}{\theta \gamma(\theta+1)}
$$


where, $\mu(\theta, \gamma)$ is the individual premium.

Now, according to the squared error loss function, the corresponding $\mathrm{BE}$ for the parameter $\theta$ and $\gamma$ is derived by substituting the posterior distribution (3.5) in (3.3), as follows:

$$
P_{S E L F}^{B}=E[\mu(\theta, \gamma) X]=\int_{0}^{\infty} \int_{0}^{\infty} \mu(\theta, \gamma) P(\theta, \gamma \mid x) d \theta d \gamma
$$

Following the procedure as discussed above, we have:

$$
\begin{aligned}
G(\theta, \gamma) & =(a-1) \log \theta+(c-1) \log \beta-(b \theta+d \beta) \\
L(\theta, \gamma) & =2 n \log \theta-n \log \gamma-n \log (\theta+1) \\
& +\sum_{i=1}^{n} \log \left[(\gamma+\gamma \theta-\theta) x_{i}+1\right]-\theta \sum_{i=1}^{n} x_{i}
\end{aligned}
$$

It may easily be verified that:

$$
\begin{array}{cl}
u_{\theta}=\frac{-\left(2 \gamma \theta^{2}+4 \gamma \theta-\theta^{2}+2 \gamma\right)}{\gamma\left(\theta+\theta^{2}\right)^{2}}, & u_{\gamma}=\frac{1}{\gamma^{2}(1+\theta)}, \\
u_{\theta \theta}=\frac{2\left(2 \gamma \theta^{3}+6 \gamma \theta^{2}+6 \gamma \theta-\theta^{3}+2 \gamma\right)}{\gamma\left(\theta+\theta^{2}\right)^{3}}, & u_{\gamma \gamma}=\frac{-2}{\gamma^{3}(1+\theta)}, \\
u_{\theta \gamma}=\frac{-\theta^{2}}{\left(\gamma\left(\theta+\theta^{2}\right)\right)^{2}}, & u_{\gamma \theta}=\frac{-1}{(\gamma(1+\theta))^{2}}
\end{array}
$$

Which are replaced in the following equation:

$$
\begin{aligned}
& P_{S E L F}^{B}=E[\mu(\hat{\theta}, \hat{\gamma}) X]=\hat{u}(\hat{\theta}, \hat{\gamma}) \\
& +\frac{1}{2}\left[\left(\hat{u}_{\gamma}+2 \hat{u}_{\gamma} \hat{p}_{\gamma}\right) \hat{\sigma}_{r \gamma}+\left(\hat{u}_{\theta \theta}+2 \hat{u}_{\theta} \hat{p}_{\theta}\right) \hat{\sigma}_{\theta \theta}\right] \\
& +\frac{1}{2}\left[\begin{array}{l}
\left(\hat{u}_{\gamma} \hat{\sigma}_{r}\right)\left(\hat{L}_{r \gamma} \hat{\sigma}_{r \gamma}+\hat{L}_{\theta \theta \gamma} \hat{\sigma}_{\theta \theta}\right) \\
\left.+\left(\hat{u}_{\theta} \hat{\sigma}_{\theta \theta}\right)\left(\hat{L}_{\theta \gamma} \hat{\sigma}_{\theta \theta}+\hat{L}_{\theta \theta \theta} \hat{\sigma}_{\theta \theta}\right)\right]
\end{array}\right.
\end{aligned}
$$

\section{On Bayesian Premium Estimators under Linear-} Exponential (Linex) Loss Function

The linear-exponential (Linex) loss function is justified by the fact that this asymmetric loss function rises approximately linearly on one side of zero and approximately exponentially on the other side) which is asymmetric, was introduced by Metiri et al. (2016), Varian (1975; Basu and Ebrahimi (1991), Pandey (1997), ), Rojo (1987), Soliman (2000) and Nassar et al. (2004).

It may be expressed as:

$$
L(\hat{\beta}, \beta)=\exp (\alpha(\hat{\beta}-\beta))-\alpha(\hat{\beta}-\beta)-1, \alpha \neq 0
$$

The sign and magnitude of the shape parameter $a$ reflect the direction and degree of asymmetry, respectively. For $a$ close to zero, the Linex loss is approximately squared error loss and therefore almost symmetric. The posterior expectation of the Linex loss function equation is:

$$
E[L(\hat{\beta}, \beta)] \propto e^{\alpha \hat{\beta}} E\left[e^{(-\alpha \beta)}\right]-\alpha(\hat{\beta}-E(\beta))-1
$$

By the result of Zellner (1986), the estimator of $\beta$ under the Linex loss $\hat{\beta}$ which minimizes the above equation is given by:

$$
\hat{\beta}=-\frac{1}{\alpha} \log \left[E\left[e^{-\alpha \beta}\right]\right]
$$

In this study, the aim is to find the Bayesian premium estimator $P_{\mathrm{LIN}}^{B}$ which is the value that minimizes the above equation, it is given by:

$$
P_{\mathrm{LIN}}^{B}=-\frac{1}{\alpha} \log \left[E\left[e^{-\alpha \mu(\theta, \gamma)}\right]\right]
$$

When the expectation $E\left[e^{-\alpha \mu(\theta, \gamma)}\right]$ exists and finite (see Calabria and Pulcini (1969)).

Thompson and Basu (1996) identified a family of loss functions $L(\Delta)$ where $\Delta$ is either the estimation error $(\hat{\beta}, \beta)$, such that

- $L(0)=0$

- $L(\Delta)>(<) L(-\Delta)>0$ for all $\Delta>0$

- $L(\cdot)$ is twice differentiable with.

- $\quad L^{\prime}(0)=0$ and $L^{\prime \prime}(\Delta)>0$ for all $\Delta \neq 0$

- $0<L^{\prime}(\Delta)>(<)-L^{\prime}(-\Delta)>0$ for all $\Delta>0$

\section{Posterior Distribution using the Gamma Prior}

The corresponding Bayesian premium estimator under the linear-exponential (Linex) loss function is:

$$
P_{\mathrm{LIN}}^{B}=-\frac{1}{\alpha} \log [E[h(\theta, \gamma) \mid X]
$$

Where:

$$
h(\theta, \gamma)=e^{-\alpha \mu(\theta, \gamma)}
$$

Now, according to the linear-exponential loss function, the corresponding Bayes estimator for the parameter $\theta$ and $\gamma$ is derived by substituting the posterior distribution (3.15) in (3.3), as follows:

$$
E\left[e^{-\alpha \mu(\theta, \gamma)} \mid X\right]=\int_{0}^{\infty} \int_{0}^{\infty} e^{-\alpha \mu(\theta, \gamma)} P(\theta, \gamma \mid x) d \theta d \gamma
$$


$L(\theta, \gamma)$ and $G(\theta, \gamma)$ are the same as those given in (3.8) and (3.7). Following the same steps explained above, we have:

$$
\begin{array}{ll}
h_{\theta}=-\alpha\left[u_{\theta} e^{-\alpha u(\theta, \gamma)}\right], & h_{\theta \theta}=-\alpha\left[\left(u_{\theta \theta}-\alpha u_{\theta}{ }^{2}\right) e^{-\alpha u(\theta, \gamma)}\right], \\
h_{\theta \gamma}=-\alpha\left[\left(u_{\theta \gamma}-\alpha u_{\theta} u_{\gamma}\right) e^{-\alpha u(\theta, \gamma)}\right], & h_{\gamma}=-\alpha\left[u_{\gamma} e^{-\alpha u(\theta, \gamma)}\right], \\
h_{\gamma \gamma}=-\alpha\left[\left(u_{\gamma \gamma}-\alpha u_{\gamma}^{2}\right) e^{-\alpha u(\theta, \gamma)}\right], & h_{\gamma \theta}=-\alpha\left[\left(u_{\gamma \theta}-\alpha u_{\theta} u_{\gamma}\right) e^{-\alpha u(\theta, \gamma)}\right.
\end{array}
$$

Which are replaced in the following equation:

$$
\begin{aligned}
& P_{\mathrm{LIN}}^{B}=-\frac{1}{\alpha} \log E\left[e^{-\alpha u(\theta, \gamma)}\right]=-\frac{1}{\alpha} \log \left[e^{-\alpha u(\hat{\theta}, \hat{\gamma})}\right. \\
& +\frac{1}{2}\left[\left(\hat{h}_{\gamma}+2 \hat{h}_{\gamma} \hat{p}_{\gamma}\right) \hat{\sigma}_{\gamma}+\left(\hat{h}_{\theta \theta}+2 \hat{h}_{\theta} \hat{p}_{\theta}\right) \hat{\sigma}_{\theta \theta}\right] \\
& +\frac{1}{2}\left[\begin{array}{l}
\left(\hat{h}_{\gamma} \hat{\sigma}_{\gamma \gamma}\right)\left(\hat{L}_{r \gamma} \hat{\sigma}_{r \gamma}+\hat{L}_{\theta \theta \gamma} \hat{\sigma}_{\theta \theta}\right) \\
+\left(\hat{h}_{\theta} \hat{\sigma}_{\theta \theta}\right)\left(\hat{L}_{\theta \gamma} \hat{\sigma}_{\theta \theta}+\hat{L}_{\theta \theta \theta} \hat{\sigma}_{\theta \theta}\right)
\end{array}\right]
\end{aligned}
$$

\section{Elicitation of Hyper Parameter}

In this work, we focus on the method proposed by Ahn et al. (2007) to determinate the hyper parameters $a, b, c$ and $d$ of the Gamma prior, this technique is based on bootstrap method. We use same steps that Garthwaite et al. (2004) and Ali et al. (2013).

\section{Simulation Study}

In simulation part, we use the Monte Carlo method for simulation study which is performed to compare the methods of estimation by using mean square Errors (MSE's) as follows:

$$
\operatorname{MSE}\left(P^{B}\right)=\sum_{i=1}^{N} \frac{\left(\hat{p}^{B}-\mu(\theta, \gamma)\right)^{2}}{N}
$$

where, $N$ is the number of replications. We generated 100000 samples of size $n=20,40,60,80,100$ and 1000 to represent small, moderate and large sample sizes from Gamma Lindley distribution with four values of $\theta(\theta=$ $0.35423,1,9)$ with $(\gamma=1.5,3,4.5)$. In order to compare the Bayesian premium estimators obtained in the above section under three different loss functions, we choose the values of the hyper parameters $a, b, c$ and $d$ as following $(a, b, c, d)=(1.2,0.001,1,0.3)$, with two values of Linex loss symmetry $(a=0.1,-0.5)$.

\section{Results and Discussion}

The performance depends on the form of the prior distribution, and the loss function assumed. Most authors used squared error as symmetric loss function. However, in practice, the real loss function is often not symmetric. It may be noted in the Gamma Lindley distribution that when $\theta$ increases $\mu(\theta, \gamma)$, decreases and the Bayesian premium estimator tends to $\mu(\theta, \gamma)$. Similarly, it has also been observed that the Bayesian premium estimator that

\begin{tabular}{|c|c|c|c|c|c|c|c|c|}
\hline \multirow[b]{2}{*}{$\gamma$} & \multirow[b]{2}{*}{$\theta$} & \multirow[b]{2}{*}{$\mu(\theta, \gamma)$} & \multicolumn{6}{|l|}{$n$} \\
\hline & & & 20 & 40 & 60 & 80 & 100 & 1000 \\
\hline \multirow{6}{*}{1.5} & 0.35423 & 5.153765 & 4.997307 & 5.071424 & $5 ., 098026$ & 5.111654 & 5.119931 & 5.150331 \\
\hline & & & $(3.0463 \mathrm{e}-05)$ & $(9.8093 \mathrm{e}-07)$ & $(9.3100 \mathrm{e}-08)$ & $(1.0252 \mathrm{e}-08)$ & $(4.4874 \mathrm{e}-10)$ & $(3.4341 \mathrm{e}-10)$ \\
\hline & 1 & 1.666667 & 1.614993 & 1.634784 & 1.644231 & 1.649422 & 1.652677 & 1.665202 \\
\hline & & & $(9.4719 \mathrm{e}-05)$ & $(3.4782 \mathrm{e}-06)$ & $(4.347 \mathrm{e}-07)$ & $(8.3599 \mathrm{e}-08)$ & $(1.8581 \mathrm{e}-08)$ & $(3.3660 \mathrm{e}-10)$ \\
\hline & $9: 0$ & 0.1555556 & 0.1673283 & 0.1566296 & 0.154794 & 0.1544417 & 0.154415 & 0.1553615 \\
\hline & & & $(3.7862 \mathrm{e}-05)$ & $(9.0926 \mathrm{e}-06)$ & $(1.8710 \mathrm{e}-06)$ & $(5.7640 \mathrm{e}-07)$ & $(2.3145 \mathrm{e}-07)$ & $(4.9876 \mathrm{e}-11)$ \\
\hline \multirow{5}{*}{3} & 0.35423 & 5.399907 & 5.245517 & 5.320573 & 5.346562 & 5.359731 & 5.367686 & 5.396656 \\
\hline & & & $(3.4648 \mathrm{e}-08)$ & $(3.3306 \mathrm{e}-07)$ & $(2.7152 \mathrm{e}-07)$ & $(1.9377 \mathrm{e}-07)$ & $(1.4095 \mathrm{e}-07)$ & $(2.0841 \mathrm{e}-09)$ \\
\hline & 1 & 1.833333 & 1.771582 & 1.800505 & 1.81104 & 1.816463 & 1.819766 & 1.831952 \\
\hline & & & $(3.5702 \mathrm{e}-07)$ & $(2.6895 \mathrm{e}-07)$ & $(2.623254 \mathrm{e}-07)$ & $(1.9752 \mathrm{e}-07)$ & $(1.4743 e-07)$ & $(2.3332 \mathrm{e}-09)$ \\
\hline & 9.0 & 0.1888889 & 0.1832947 & 0.1849694 & 0.186032 & 0.1866555 & 0.1870587 & 0.1886903 \\
\hline \multirow{7}{*}{4.5} & & & $(3.9372 \mathrm{e}-06)$ & $(2.1432 \mathrm{e}-07)$ & $(3.8176 \mathrm{e}-08)$ & $(1.0968 \mathrm{e}-08)$ & $(4.0807 \mathrm{e}-09)$ & $(4.6508 \mathrm{e}-13)$ \\
\hline & 0.35423 & 5.481954 & 5.329274 & 5.403857 & 5.429509 & 5.442479 & 5.450306 & 5.478765 \\
\hline & & & $(1.1682 \mathrm{e}-06)$ & $(9.6732 \mathrm{e}-07)$ & $(5.5784 \mathrm{e}-07)$ & $(3.52083 \mathrm{e}-07)$ & $(2.4046 \mathrm{e}-07)$ & $(2.9733 \mathrm{e}-09)$ \\
\hline & 1 & 1.888889 & 1.827011 & 1.856448 & 1.866937 & 1.872305 & 1.875564 & 1.887536 \\
\hline & & & $(6.2464 \mathrm{e}-07)$ & $(9.0892 \mathrm{e}-07)$ & $(5.6647 \mathrm{e}-07)$ & $(3.6896 \mathrm{e}-07)$ & $(2.5635 \mathrm{e}-07)$ & $(3.3429 \mathrm{e}-09)$ \\
\hline & 9.0 & 0.2 & 0.193616 & 0.1959277 & 0.1970839 & 0.1977365 & 0.1981523 & 0.1998017 \\
\hline & & & $(2.0774 \mathrm{e}-06)$ & $(1.0706 \mathrm{e}-07)$ & $(1.682 \mathrm{e}-08)$ & $(4.0851 \mathrm{e}-09)$ & $(1.2295 \mathrm{e}-09)$ & $(5.3703 \mathrm{e}-12)$ \\
\hline
\end{tabular}
gives the smallest average absolute error over all the other Bayesian premium estimators in majority of the cases is Bayesian premium estimator under the generalized prior with Linex loss function especially when the loss parameter is less than zero i.e., $(a=-0.5)$.

Table 1. Bayesian premium estimators and respective MSE's under squared error loss function $(a=1, b=0.04, c=1, d=0.04)$ 
Table 2. Bayesian premium estimators and respective MSE's under LINEX loss function $(a=2, b=0.25, c=2, d=0.5, a=0.1)$ $n$

\begin{tabular}{|c|c|c|c|c|c|c|c|c|}
\hline$\gamma$ & $\theta$ & $\mu(\theta, \gamma)$ & 20 & 40 & 60 & 80 & 100 & 1000 \\
\hline \multirow{3}{*}{1.5} & 0.35423 & 5.153765 & $\begin{array}{l}4.96047 \\
(0.0373628)\end{array}$ & $\begin{array}{l}5.052458 \\
(0.0102630)\end{array}$ & $\begin{array}{l}5.085255 \\
(0.00469350)\end{array}$ & $\begin{array}{l}5.102028 \\
(0.00267671)\end{array}$ & $\begin{array}{l}5.112207 \\
(0.00172703)\end{array}$ & $\begin{array}{l}5.14955 \\
(1.7764 \mathrm{e}-05)\end{array}$ \\
\hline & 1 & 1.666667 & $\begin{array}{l}1.61492 \\
(0.00267783)\end{array}$ & $\begin{array}{l}1.634389 \\
(0.0010418)\end{array}$ & $\begin{array}{l}1.643829 \\
(0.000521554)\end{array}$ & $\begin{array}{l}1.649062 \\
(0.000309914)\end{array}$ & $\begin{array}{l}1.65236 \\
(0.000204693)\end{array}$ & $\begin{array}{l}1.665159 \\
(2.2741 \mathrm{e}-06)\end{array}$ \\
\hline & 9.0 & 0.1555556 & $\begin{array}{l}0.1672811 \\
(0.00013749)\end{array}$ & $\begin{array}{l}0.1566093 \\
(1.1104 \mathrm{e}-06)\end{array}$ & $\begin{array}{l}0.1547803 \\
(6.0098 \mathrm{e}-07)\end{array}$ & $\begin{array}{l}0.1544313 \\
(1.2640 \mathrm{e}-06)\end{array}$ & $\begin{array}{l}0.154334 \\
(1.4922 \mathrm{e}-06)\end{array}$ & $\begin{array}{l}0.1553606 \\
(3.8000 \mathrm{e}-08)\end{array}$ \\
\hline \multirow{3}{*}{3} & 0.35423 & 5.399907 & $\begin{array}{l}5.207548 \\
(0.0370017)\end{array}$ & $\begin{array}{l}5.301051 \\
(0.00977239)\end{array}$ & $\begin{array}{l}5.333425 \\
(0.00441983)\end{array}$ & $\begin{array}{l}5.349831 \\
(0.00250760)\end{array}$ & $\begin{array}{l}5.348782 \\
(0.00261379)\end{array}$ & $\begin{array}{l}5.394745 \\
(2.6644 \mathrm{e}-05)\end{array}$ \\
\hline & 1 & 1.833333 & $\begin{array}{l}1.766747 \\
(0.00443374)\end{array}$ & $\begin{array}{l}1.798005 \\
(0.00124808)\end{array}$ & $\begin{array}{l}1.809354 \\
(0.000575029)\end{array}$ & $\begin{array}{l}1.815191 \\
(0.000329127)\end{array}$ & $\begin{array}{l}1.818745 \\
(0.000212811)\end{array}$ & $\begin{array}{l}1.831848 \\
(2.2056 \mathrm{e}-06)\end{array}$ \\
\hline & 9.0 & 0.1888889 & $\begin{array}{l}0.1832283 \\
(3.2042 \mathrm{e}-05)\end{array}$ & $\begin{array}{l}0.1849343 \\
(1.5639 \mathrm{e}-05)\end{array}$ & $\begin{array}{l}0.1860081 \\
(8.2986 \mathrm{e}-06)\end{array}$ & $\begin{array}{l}0.1866374 \\
(5.0693 \mathrm{e}-06)\end{array}$ & $\begin{array}{l}0.1870441 \\
(3.4030 \mathrm{e}-06)\end{array}$ & $\begin{array}{l}0.1886888 \\
(4.1002 \mathrm{e}-08) \backslash\end{array}$ \\
\hline \multirow{3}{*}{4.5} & 0.35423 & 5.481954 & $\begin{array}{l}5.290906 \\
(0.0364993)\end{array}$ & $\begin{array}{l}5.384137 \\
(0.00956830)\end{array}$ & $\begin{array}{l}5.416239 \\
(0.00431850)\end{array}$ & $\begin{array}{l}5.43248 \\
(0.00244773)\end{array}$ & $\begin{array}{l}5.442285 \\
(0.00157368)\end{array}$ & $\begin{array}{l}5.477955 \\
(1.5994 \mathrm{e}-05)\end{array}$ \\
\hline & 1 & 1.888889 & $\begin{array}{l}1.865187 \\
(0.000561776)\end{array}$ & $\begin{array}{l}1.870985 \\
(0.00032056)\end{array}$ & $\begin{array}{l}1.874504 \\
(0.000206913)\end{array}$ & $\begin{array}{l}1.879245 \\
(9.3002 \mathrm{e}-05)\end{array}$ & $\begin{array}{l}1.88404 \\
(2.3511 \mathrm{e}-05)\end{array}$ & $\begin{array}{l}1.887429 \\
(2.1324 \mathrm{e}-06)\end{array}$ \\
\hline & 9.0 & 0.2 & $\begin{array}{l}0.1935402 \\
(4.1729 \mathrm{e}-05)\end{array}$ & $\begin{array}{l}0.1958878 \\
(1.6909 \mathrm{e}-05)\end{array}$ & $\begin{array}{l}0.1970568 \\
(8.6622 \mathrm{e}-06)\end{array}$ & $\begin{array}{l}0.197716 \\
(5.2166 \mathrm{e}-06)\end{array}$ & $\begin{array}{l}0.1981358 \\
(3.4754 \mathrm{e}-06)\end{array}$ & $\begin{array}{l}0.1998001 \\
(3.9978 \mathrm{e}-08)\end{array}$ \\
\hline
\end{tabular}

Table 3. Bayesian premium estimators and respective MSE's under LINEX loss function $(a=2, b=0.25, c=2, d=0.5, a=-0.5)$

\begin{tabular}{|c|c|c|c|c|c|c|c|c|}
\hline \multirow[b]{2}{*}{$\gamma$} & \multirow[b]{2}{*}{$\theta$} & \multirow[b]{2}{*}{$\mu(\theta, \gamma)$} & \multicolumn{6}{|l|}{$n$} \\
\hline & & & 20 & 40 & 60 & 80 & 100 & 1000 \\
\hline & 0.35423 & 5.153765 & $\begin{array}{l}5.171916 \\
(0.000329548)\end{array}$ & $\begin{array}{l}5.163393 \\
(9.2716 \mathrm{e}-05)\end{array}$ & $\begin{array}{l}5.160425 \\
(4.4359 \mathrm{e}-05)\end{array}$ & $\begin{array}{l}5.158868 \\
(2.6042 \mathrm{e}-05)\end{array}$ & $\begin{array}{l}5.157903 \\
(1.7130 \mathrm{e}-05)\end{array}$ & $\begin{array}{l}5.154202 \\
(1.9100 \mathrm{e}-07)\end{array}$ \\
\hline \multirow[t]{3}{*}{1.5} & 1 & 1. 666667 & $\begin{array}{l}1.635624 \\
(0.000963748)\end{array}$ & $\begin{array}{l}1.64558 \\
(0.000444644)\end{array}$ & $\begin{array}{l}1.65154 \\
(0.000228809)\end{array}$ & $\begin{array}{l}1.654946 \\
(0.000137363)\end{array}$ & $\begin{array}{l}1.657117 \\
(9.1198 \mathrm{e}-05)\end{array}$ & $\begin{array}{l}1.665653 \\
(1.0270 \mathrm{e}-06)\end{array}$ \\
\hline & 9.0 & 0.1555556 & $\begin{array}{l}0.1675587 \\
(0.000144080)\end{array}$ & $\begin{array}{l}0.1567301 \\
(1.3795 \mathrm{e}-06)\end{array}$ & $\begin{array}{l}0.1548624 \\
(4.8045 \mathrm{e}-07)\end{array}$ & $\begin{array}{l}0.1544938 \\
(1.1273 \mathrm{e}-06)\end{array}$ & $\begin{array}{l}0.1544571 \\
(1.2066 \mathrm{e}-06)\end{array}$ & $\begin{array}{l}0.1553659 \\
(3.5972 \mathrm{e}-08)\end{array}$ \\
\hline & 0.35423 & 5.399907 & $\begin{array}{l}5.375009 \\
(0.000619920)\end{array}$ & $\begin{array}{l}5.389093 \\
(0.000116943)\end{array}$ & $\begin{array}{l}5.393134 \\
(4.5865 \mathrm{e}-05)\end{array}$ & $\begin{array}{l}5.395002 \\
(2.4053 \mathrm{e}-05)\end{array}$ & $\begin{array}{l}5.39607 \\
(1.4720 \mathrm{e}-05)\end{array}$ & $\begin{array}{l}5.399556 \\
(1.2290 \mathrm{e}-07)\end{array}$ \\
\hline \multirow[t]{3}{*}{3} & 1 & 1.833333 & $\begin{array}{l}1.824958 \\
(7.0140 \mathrm{e}-05)\end{array}$ & $\begin{array}{l}1.828867 \\
(1.9947 \mathrm{e}-05)\end{array}$ & $\begin{array}{l}1.83034 \\
(8.9579 \mathrm{e}-06)\end{array}$ & $\begin{array}{l}1.83109 \\
(5.0335 \mathrm{e}-06)\end{array}$ & $\begin{array}{l}1.831541 \\
(3.2128 \mathrm{e}-06)\end{array}$ & $\begin{array}{l}1.833156 \\
(3.1410 \mathrm{e}-08)\end{array}$ \\
\hline & $9: 0$ & 0.1888889 & $\begin{array}{l}0.1886775 \\
(4.4712 \mathrm{e}-08)\end{array}$ & $\begin{array}{l}0.1878023 \\
(1.1807 \mathrm{e}-06)\end{array}$ & $\begin{array}{l}0.1879242 \\
(9.3055 \mathrm{e}-07)\end{array}$ & $\begin{array}{l}0.1880724 \\
(6.6671 \mathrm{e}-07)\end{array}$ & $\begin{array}{l}0.1881903 \\
(4.8805 \mathrm{e}-07)\end{array}$ & $\begin{array}{l}0.1886978 \\
(3.65251 \mathrm{e}-09)\end{array}$ \\
\hline & 0.35423 & 5.481954 & $\begin{array}{l}5.512915 \\
(0.000958594)\end{array}$ & $\begin{array}{l}5.500253 \\
(0.000334847)\end{array}$ & $\begin{array}{l}5.494853 \\
(0.000166383)\end{array}$ & $\begin{array}{l}5.491902 \\
(9.8955 \mathrm{e}-05)\end{array}$ & $\begin{array}{l}5.490047 \\
(6.5484 \mathrm{e}-05)\end{array}$ & $\begin{array}{l}5.482814 \\
(7.384 \mathrm{e}-07)\end{array}$ \\
\hline \multirow[t]{2}{*}{4.5} & 1 & 1.888889 & $\begin{array}{l}1.852012 \\
(0.00135993)\end{array}$ & $\begin{array}{l}1.869397 \\
(0.000379915)\end{array}$ & $\begin{array}{l}1.875674 \\
(0.00017463)\end{array}$ & $\begin{array}{l}1.878897 \\
(9.9843 \mathrm{e}-05)\end{array}$ & $\begin{array}{l}1.880857 \\
(6.4515 \mathrm{e}-05)\end{array}$ & $\begin{array}{l}1.888072 \\
(6.6713 \mathrm{e}-07)\end{array}$ \\
\hline & 9.0 & 0.2 & $\begin{array}{l}0.193994 \\
(3.6067 \mathrm{e}-05)\end{array}$ & $\begin{array}{l}0.196127 \\
(1.4998 \mathrm{e}-05)\end{array}$ & $\begin{array}{l}0.197219 \\
(7.7314 \mathrm{e}-06)\end{array}$ & $\begin{array}{l}0.197839 \\
(4.6692 \mathrm{e}-06)\end{array}$ & $\begin{array}{l}0.198234 \\
(3.1156 \mathrm{e}-06)\end{array}$ & $\begin{array}{l}0.199810 \\
(3.6028 \mathrm{e}-08)\end{array}$ \\
\hline
\end{tabular}

It is always followed by efficiency by the squared error loss as shown in Table 1 and 2. According to Table 3, it can be shown that the Bayesian premium estimator under entropy loss function is also more efficient than the Bayes estimator under squared error and Linex loss only in the case of the high values of $\theta$. As the sample size increases, it is observed that all the estimators average MSEs almost tends to $\mu(\theta, \gamma)$. Furthermore, we conclude that the performance is approximately equal with smaller posterior risk. Also, results of the Gamma Lindley prior are more precise usually under Linex $(a=-0.5)$. From the above-mentioned discussion, we may conclude that the Bayes procedure discussed in this paper can be recommended for their use.

\section{Conclusion}

This paper treat the problem of Bayesian premium estimators for the Gamma Lindley distribution, under asymmetric and symmetric loss functions, the risk parameter for a policyholder is never known in reality, we constructed Bayesian premium estimators following Bayesian inference techniques. By imposing a prior 
distribution on, we are able to describe the risk structure for the entire rating class. In practice, the choice of this prior distribution is subjective to personal judgments or induced from historical data of the corresponding groups. Using numerical simulation, it seems that the Bayesian premiums are consistent and verifies the condition of convergence to the individual premium. From the results, we observe that in most cases, Bayesian premium estimators for the Gamma Lindley distribution is more effective because it converges faster to the individual premium usually under Linex $(a=-0.5)$ which have been mentioned in the tables above. For future studies, we can consider the distributions of inverse Lindley, inverse Gamma-Lindley as conditional distributions instead of Zeghdoudi distribution, under entropy, Linex and squared error loss functions respectively. In addition, this work can be extended using censored data.

\section{Acknowledgment}

The authors acknowledge Editor-in-Chief, Dr.Carlo Bianca, of this journal for the constant encouragement to finalize the paper.

\section{Author's Contributions}

Ahmed Sadoun: Participated in simulation part and writing the paper.

Halim Zeghdoudi: Designed the research plan, organized the study and writing the paper.

Fatma Zohra Attoui: Participated in simulation part.

Mohamed Riad Remita: Participated in writing the paper.

\section{Ethics}

This paper is original and contains unpublished material. The corresponding author confirms that all of the other authors have read and approved the manuscript and no ethical issues involved.

\section{References}

Ahn, S.E., C.S. Park and H.M. Kim, 2007. Hazard rate estimation of a mixture model with censored lifetimes, Stoch. Environ. Res. Assess., 21: 711-716. DOI: 10.1007/s00477-006-0082-1

Ali, S., M. Aslam, S. Mohsin and A. Kazmi, 2013. A study of the effect of the loss function on Bayes Estimate, posterior risk and hazard function for Lindley distribution. Applied Math. Modelling, 37: 6068-6078. DOI: 10.1016/j.apm.2012.12.008

Bailey, A.L., 1950. Credibility procedures, Laplace's generalization of Bayes' rule and the combination of collateral knowledge with observed data. Proce. Casualty Actuarial Society, 37: 7-23, 1950.
Basu, A.P. and N. Ebrahimi, 1991. Bayesian approach to life testing and reliability estimation using asymmetric loss function, J. Statist. Plann. Infer., 29: 21-31. DOI: 10.1016/0378-3758(92)90118-C

Bühlmann, H. and E. Straub, 1996. Glaubgwürdigkeit für Schadensutze. Bulletin Swiss Association Actuaries, 70: 111-133.

Calabria, R. and G. Pulcini, 1969. Point estimation under asymmetric loss functions for left truncated exponential samples. Comm. Statist. Theory Methods, 25: 585-600. DOI: $10.1080 / 03610929608831715$

Dey, D., M. Ghosh and W.E. Strawderman, 1999. On estimation with balanced loss functions. Statistics Probability Lett., 45: 97-101. DOI: /10.1016/S0167-7152(99)00047-4

Dey, D.K. and P.L. Liu, 1992. On comparison of estimators in a generalized life model. Microelectron Reliab., 32: 207-221. DOI: 10.1016/0026-2714(92)90099-7

Garthwaite, H.P., B.J. Kadane and A. O'Hagan, 2004. Elicitation. University of Sheffield.

Ghitany, M.E. and D.K. Al-Mutairi, 2009. Estimation methods for the discrete Poisson-Lindley distribution. J. Statistical Computation Simulation, 79: 1-9. DOI: 10.1080/00949650701550259

Ghitany, M.E., B. Atieh and S. Nadarajah, 2008. Lindley distribution and its application. Math. Comput. Simulation, 78: 493-506. DOI: $10.1016 /$ j.matcom.2007.06.007

Howlader, H.A. and A. Hossain, 2002. Bayesian survival estimation of Pareto distribution of the second kind based on failure censored data. Computational Statistics Data Anal., 38: 301-314. DOI: 10.1016/S0167-9473(01)00039-1

Ibrahim, N.A., C.B. Guure and A.O.M. Ahmed, 2012. Bayesian estimation of two-parameter weibull distribution using extension of Jeffreys' prior information with three loss functions. Mathematical Problems Eng., 2012: 1-13. DOI: $10.1155 / 2012 / 589640$

Jaheen, Z.F., 2005. On record statistics from a mixture of two exponential distributions. J. Statist. Comput. Simul., 75, 1-11. DOI: 10.1080/00949650410001646924

Krishna, H. and K. Kumar, 2011. Reliability estimation in Lindley distribution with progressively type II right censored sample. Math. Comput. Simul., 82: 281-294. DOI: 10.1016/j.matcom.2011.07.005

Legendre, A., 1805. Nouvelles méthodes pour la determination des orbites des comètes, Courcier, City Paris. 
Lindley, D.V., 1958. Fiducial distributions and Bayes' theorem. J. Royal Statistical Society, Series A20: 102-107.

Lindley, D.V., 1980. Approximate Bayes methods. Trabajos de Estadistica de Investigacion Operativa, 31: 223-245. DOI: $10.1007 / \mathrm{BF} 02888353$

Metiri, F., H. Zeghdoudi and M.R. Remita, 2016. On bayes estimates of lindley distribution under linex loss function: Informative and non informative priors. Global J. Pure Applied Math., 12: 391-400.

Nassar, M. and F.H. Eissa, 2004. Bayesian estimation for the exponentiated Weibull model. Commun Statistical theory Meth., 33: 2343-2362. DOI: $10.1081 / \mathrm{STA}-200031447$

Pandey, B.N., 1997. T-estimator of the scale parameter of the exponential distribution using Linex loss function. Commun. Statistical Theory. Meth., 26: 2191-2202. DOI: 10.1080/03610929708832041

Rojo. 1987. On the admissibility of "cx $+d$ " with respect to the Linex loss Function, Commun. Statistical Theory Meth., 116: 3745-3748.

Sankaran, M., 1970. The discrete Poisson-Lindley distribution. Biometrics, 26: 145-149. DOI: $10.2307 / 2529053$

Shanker, R., S. Sharma and R. Shanker, 2013. A twoparameter lindley distribution for modeling waiting and survival times data. Applied Math., 4: 1-6. DOI: 10.4236/am.2013.42056

Soliman, A., 2000. Comparison of Linex and quadratic Bayes estimators for the Rayleigh distribution. Commun. Statist. Theor. Meth., 29: 95-107. DOI: $10.1080 / 03610920008832471$
Thompson, R.D. and A.P. Basu, 1996. Asymmetric loss function for estimating reliability. J. Bayesian Anal. Statistics Econometrics.

Varian, H.R., 1975. A Bayesian approach to real estate assessment. Studies in Bayesian Econometrics and Statistics, Amsterdam: North-Holland, pp: 195-208.

Zeghdoudi, H. And Nedjar, S, 2016. Gamma Lindley distribution and its application. J. Applied Probability Statistics, 11: 129-138.

Zeghdoudi, H. and S. Nedjar, 2016a. On Gamma Lindley distribution: proprieties and simulation. J. Comput. Applied Math., 298: 167-174. DOI: 10.1016/j.cam.2015.11.047

Zeghdoudi, H. and N. Lazri, 2016. On Lindley-Pareto distribution: properties and application. J. Math. Stat. Operations Res., 3: 1-7.

Zeghdoudi, H. and S. Nedjar, 2016b. A Pseudo Lindley distribution and its application, J. Afri. Statistika, 11: 923-932. DOI:10.16929/as/2016.923.83

Zeghdoudi, H. and S. Nedjar, 2017. On pseudo Lindley distribution: Properties and applications. J. New Trends Math. Sci., 5: 59-65. DOI: $10.20852 /$ ntmsci.2017.126

Zeghdoudi, H. and S. Nedjar, 2017a. A Poisson pseudo Lindley distribution and its application, J. Probability Statistical Sci., 15: 19-28.

Zellner, A., 1986. Bayesian estimation and prediction using asymmetric loss functions. J. Amsterdam Statistical Association, 81: 446-451. 